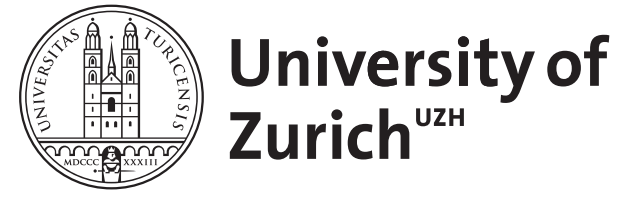

\title{
Andreas Kraß, Geschriebene Kleider. Höfische Identität als literarisches Spiel. 2006
}

\author{
Zimmermann, Julia
}

DOI: https://doi.org/10.1515/arbi.2008.086

Posted at the Zurich Open Repository and Archive, University of Zurich ZORA URL: https://doi.org/10.5167/uzh-154617

Journal Article

Published Version

Originally published at:

Zimmermann, Julia (2008). Andreas Kraß, Geschriebene Kleider. Höfische Identität als literarisches Spiel. 2006. Arbitrium: Zeitschrift für Rezensionen zur germanistischen Literaturwissenschaft, 26(3):267274.

DOI: https://doi.org/10.1515/arbi.2008.086 
Andreas Kraß, Geschriebene Kleider. Höfische Identität als literarisches Spiel. (Bibliotheca Germanica 50) Francke, Tübingen - Basel 2006. 4 I9 S., $€$ 78,-.

Wie sich die ritterlich-höfische Gesellschaft des I 2. und I 3. Jahrhunderts des Zeichensystems der Kleidung bedient, um ihre Identität - in einer kulturwissenschaftlich orientierten literaturwissenschaftlichen Untersuchung verstanden nicht als essentielle Gegebenheit, sondern als „Produkt und Effekt eines narrativen Prozesses“ (S. 23) - zu modellieren, dieser Frage geht die an der Ludwig-Maximilians-Universität München 2002/2003 angenommene Habilitationsschrift von Andreas Kraß nach.

Weil unterschiedliche gesellschaftliche Bedeutungen von Kleidung zu erkennen geben, daß hier imaginäre Besetzungen wirksam sind, stellt Andreas $\mathrm{Kra}$ den Auseinandersetzungen mit den mittelalterlichen Texten eine Erörterung der kultur-, gesellschafts- und literaturtheoretischen Dimensionen des vestimentären Codes voran: Indem er mit Castoriades und Iser die gesellschaftstheoretischen und literaranthropologischen Perspektiven in Roland Barthes' kultursemiotisches Konzept des vestimentären Codes einarbeitet, schafft Kraß sich sein methodisches Instrumentarium für weitangelegte Analysen, die der Vielfalt kultureller Bedeutungen von Kleidung in höfischen Erzählungen des deutschsprachigen Mittelalters nachgehen.

Im ersten von insgesamt vier Hauptteilen der Studie widmet sich Kraß den anthropologischen und narratologischen Spielräumen des vestimentären Codes. Die christliche Anthropologie der Kleidung stellt den Bezugsrahmen für ihr „laikales Gegenstück" (S. 62), für die höfische Anthropologie der Kleidung.

Aus diesem Grund zeigt Kraß anhand eingehender Analysen von spätantiken und mittelalterlichen Genesiskommentaren zunächst, wie der Sündenfall ,jene Urszene darstellt, aus der sich auch das kulturgeschichtliche, moraltheologische und ästhetische Verhältnis des höfischen Menschen zur Kleidung herleiten läßt“ (S. 28). Im Hinblick auf die angesprochene christliche Anthropologie der Kleidung sind dabei mit Kraß zwei Gewänder zu unterscheiden: das irdische Gewand des gefallenen, sündigen homo terrestris und das himmlische Gewand des von Christus erlösten, im Zustand der Gnade sich befindenden bomo caelestis. Ebenso wie sich in diesem Kontext das irdische Gewand nach dem Sündenfall paradoxerweise als Zeichen moralischer Nacktheit verstehen läßt, so läßt sich umgekehrt auch die unschuldige prälapsale Nacktheit von Adam und Eva als himmlisches Gnadenkleid sowie als Gewand der Unsterblichkeit und Engelhaftigkeit interpretieren. Das im Sündenfall verlorene Gnadenkleid kann freilich im Sakrament der Taufe (bei der Christus gleichsam als Kleid angelegt wird) wiedererlangt werden. Vor diesem Hintergrund erörtert Kraß des weiteren einerseits die Bedeutung von Mode für die höfische Gesellschaft, die ihren Status - verstanden als spezifischen Gnadenstand höfischer saelde - immer wieder auch mit dem Glanz der Kleidung ins rechte Licht zu rücken trachtet, und andererseits die klerikale Kritik, die diesen Kleiderprunk als Sünde der Eitelkeit brandmarkt. Wenn, wie Kraß festhält, die Kleriker zudem beklagen, „daß die weltliche Hofgesellschaft mit ihren strahlenden Seidenkleidern den Eindruck erwecke, sie habe die Gnade Gottes nie verloren und stehe nicht im Bann des Sündenfalls“ (S. 62), so wäre gerade in diesem Vorwurf der Diskrepanz zwischen Schein und Sein ein interessanter Ansatzpunkt für die Frage, inwiefern die geistliche Kleiderkritik diese höfischen „Verkleidungen“ tatsächlich als solche problematisiert (freilich nicht, wie Kraß es später in anderem Kontext unternimmt, als Spiel mit einer sozialen oder personalen, sondern eben gleichsam als Anschein einer ethischen Identität).

Weil Allegorese und Paradigmatik, diese „fundamentalen Typen der literarischen Nutzung des vestimentären Codes“ (S. 66), noch in der höfischen Epik im Kontext einer „Narratologie der Kleidung" stehen, demonstriert Kraß fürderhin an zwei Beispielen, wie diese beiden 
narratologischen Verfahren bereits in der althochdeutschen Bibelepik verwendet werden können. Während Otfrid von Weißenburg etwa in der Passions- und Auferstehungsgeschichte seines Evangelienbuchs vestimentäre Motive zwar zur exegetischen Deutung, nicht aber zur paradigmatischen Verdichtung der Erzählstruktur nutze, seien die Kleidermotive in der Altdeutschen Genesis durchaus so in den Handlungsfluß integriert, daß sich auch Bezüge zwischen den jeweiligen Szenen ergeben würden. Als „kardinale vestimentäre Figuren“ (S. 84) bezeichnet Kraß in diesem Zusammenhang Akte der Investitur und der Maskerade: Die Figur der Investitur diene der Affirmation des sozialen, religiösen oder politischen Standes einer Person, die Maskerade stelle hingegen eine transgressive vestimentäre Figur dar, denn hier werde Identität nicht bestätigt oder gefestigt, sondern verschleiert. Im Gregorius Hartmanns von Aue, den Kraß als drittes Beispiel folgen läßt, würden schließlich die beiden literarischen Typen Allegorese und Paradigmatik und die vestimentären Motive (Investitur/Devestitur) in einem Text souverän verwendet wiederkehren, „der seiner Gattung nach zwischen Hagiographie und höfischem Roman vermittelt" (S. 92).

Der zweite Hauptteil der Untersuchung befaßt sich sodann mit vestimentären „Spielregeln“, die der Produktion und Affirmation höfischer Identität dienen, „, weil sie unter je verschiedenem Blickwinkel Identität als feste Größe konzipieren, die man wie Kleider erwerben, zur Schau stellen und wieder verlieren kann" (S. 28). In diesem Kontext unterscheidet Kraß zwischen Investitur als Code der Einsetzung, Devestitur als Code der Aussetzung. und Epiphanie als Code der Verklärung höfischer Identität.

Die Überlegungen zur Investitur setzen sich mit den wechselseitig aufeinander bezogenen vestimentären Riten der Tauf- und Krönungsliturgie auseinander, da diese den literarischen Darstellungen von ritterlichen Einkleidungen in zweifacher Weise als Vorbild dienen: „mimetisch als narrative Inszenierungen von Tauf- und Krönungshandlungen und symbolisch als strukturelles und motivisches Substrat fiktionaler Einkleidungsszenen, die nicht direkt auf Tauf- und Krönungshandlungen referieren“ (S. 93).

In fünf Einzelanalysen widmet sich Kraß daraufhin ritterlichen Investiturszenen in den Artusromanen Erec, Irwein, Parzival und Wigalois sowie in dem Heldenepos Wolfdietrich, „um exemplarisch ein Spektrum verschiedener ritterlicher Identitätskonzepte vorzuführen“ (S. 29). Hier gelangt Kraß zu dem Befund, daß die Einkleidungsszenen in den frühen Artusromanen das Telos ritterlichen Aufstiegs markieren; das geistliche Modell werde für die spezifisch höfisch-laikale Identitätskonzeption profaniert. Im Parzival trage die Reihe von insgesamt neun Einkleidungsszenen den Erzählprozeß mit; in der Abfolge von Devestitur, $\mathrm{Bad}$ und Investitur würde insbesondere die durch Gurnemanz unternommene Einkleidung Parzivals einen „tiefgreifenden Identitätswechsel“ (S. I 28) markieren, eine Initiation, bei der der Held sich vom Narren zum Ritter wandle. Investitur sei im Parzival folglich nicht Resultat, sondern Antizipation höfischer Erziehung. Interessant scheint in der detaillierten Analyse des Parzival (stets im Vergleich zu den Einkleidungsszenen bei Chrétien) die Unterteilung der neun immer wieder aufeinander bezogenen Investiturszenen in drei verschiedene Kategorien: Die erste Kategorie umfaßt Einkleidungen durch Erzieher (etwa durch Gurnemanz oder Trevrizent, von dem Parzival eine Art Büßerrock empfängt); die zweite Kategorie enthält Mantelgaben als Indikatoren von Verpflichtungsverhältnissen (Condwiramurs, Repanse de Schoye, Cunneware), und die dritte Kategorie umfaßt zwei Szenen, in denen Parzival und Gawan beziehungsweise Feirefiz als Spiegel- und Stellvertreterfiguren mit identischen Gewändern ausgestattet werden. Getrübt wird die Freude an der Analyse lediglich durch kleinere Unstimmigkeiten: daß etwa das Motiv exotischer Kleidung dem Gralsbereich vorbehalten sei (S. I28, Anm. I05), läßt sich im Parzival sicher nicht in dieser Pauschalität bestätigen, und auch der abschließende Hinweis auf die Taufe des Feirefiz, die nach Kraß ,in Übereinstimmung mit der mittelalterlichen Taufliturgie geschildert wird“ (S. I I I), wirft Fragen auf, ist es doch gerade diese Taufszene, in der das religiöse Motiv dem ungestümen 
Liebesverlangen des Feirefiz so deutlich nachgestellt ist, daß das christliche Ritual der Taufe fast wie eine Burleske anmutet. ${ }^{1}$

Während Wolfram im Kontext der Identitätsfindung Parzivals die Variante der aufgegebenen Identität einbringt, ist es im Wigalois eine vorgegebene Identität, die nach Kraß zum Tragen komme, weil ritterliche Idealität hier als gegeben und unverlierbar inszeniert sei. Das Motiv der Investitur trete deshalb zugunsten des Rüstungsmotivs zurück, da der krisenlose Held auf seinem Weg bereits eine stabile Identität aufweise. „Mit der Akzentverschiebung von der Kleidung auf die Rüstung des Protagonisten geht nicht nur eine Revision des ritterlichen Identitätskonzepts, sondern auch eine zunehmende Betonung der Geschlechterdifferenz einher" (S. I 38), denn anders als in den früheren Artusromanen sei Kleidung nun weiblich codiert. Im Wolfdietrich schließlich zeige sich, wie auch der vestimentäre Code an der Tendenz des Heldenepos teilhabe, die ritterlichen Identitätsmodelle des Artusromans zu dekonstruieren. Trotz des kurzen Verweises auf die Nähe zwischen höfischem Roman und dem Wolfdietrich als Heldendichtung fügt sich letztgenannte nicht dem vorangestellten, exemplarisch analysierten Textensemble, das Kraß seinen Überlegungen zur Investitur zugrunde legt. Zu hinterfragen bleibt zumindest, ob die vielfach angesprochene „ritterliche Identität“ mit Blick auf den Wolfdietrich - auch bei der proklamierten Nähe zwischen Artus- und Heldenepos nicht vielmehr als eine heroische Identität problematisiert werden müßte.

Anders als die Investitur, die den Übergang von einer alten in eine neue Phase von Identität markiert, ist die auf einem metaphysischen Schönheitskonzept beruhende Epiphanie von ereignishaftem Charakter. Vor dem Hintergrund eines spezifischen Verständnisses von saelde lassen sich dabei mit Kraß die literarischen Inszenierungen von höfischer Mode „als vestimentäres Symbol für die Verklärung der höfischen Identität verstehen“ (S. I I). Gnadenhaftes Charisma gebe sich in den auratisch anmutenden Schönheitsbeschreibungen unter anderem im vestimentären Merkmal der Leuchtkraft zu erkennen. Ausgehend von diesen Annahmen befaßt sich Kraß mit seinen Überlegungen zur „Verklärung höfischer Identität“ zunächst mit dem höfischen Kleiderkult, der mit Textbeispielen zur klerikalen Kleiderkritik konfrontiert wird.

Sodann folgen erneut fünf exemplarische Analysen: Beleuchtet werden der Auftritt Enites vor der Artusgesellschaft im Erec, die (intertextuell auf den Erec verweisende) Szene, in der Siegfried im Nibelungenlied zum ersten Mal Kriemhild erblickt, die körperliche und vestimentäre Schönheitsbeschreibung Engeltruts im Engelhard, die entsprechend als Epiphanie in Szene gesetzte Schönheitsbeschreibung Parzivals bei Wolfram sowie das Motiv der Epiphanie, das im ersten Teil des Tristan die personale Union der Liebenden in ihrer ästhetischen Harmonie aufzeigt. Wenig überraschend läßt sich dabei mit Kraß festhalten, daß Reflexe auf die geistliche Kleiderkritik in den frühen Dichtungen noch deutlich erkennbar sind, während sich die späteren Fallbeispiele von den klerikalen Vorwürfen emanzipieren. Zudem sei auffallend, so Kraß, daß die vestimentären Schönheitsbeschreibungen vorwiegend auf weibliche Figuren bezogen werden, während die Investiturszenen den männlichen Protagonisten vorbehalten bleiben.

Die Devestitur nicht als vorausgehenden Teilakt, sondern als Reversion der Investitur durch freiwillige oder unfreiwillige Abdankung beziehungsweise Absetzung steht im Zentrum des darauffolgenden Teilkapitels. In

\footnotetext{
${ }^{\mathrm{I}}$ Vgl. Joachim Bumke, „Parzival und Feirefiz - Priester Johannes - Loherangrin“. In: Deutsche Vierteljabrsschrift für Literaturwissenschaft und Geistesgeschichte 65 (I99I), S. 242 245 , der auch auf die „ernste Seite“ dieser Burlesken verweist. Karl Bertau, Über Literaturgeschichte. Literarischer Kunstcharakter und Geschichte in der höfischen Epik um I200. München 1983, S. 68, hat dem Schluß des Parzival sogar den Charakter einer „geradezu betrunkenen Opernhaftigkeit" zugesprochen.
} 
diesem stellt Kraß zunächst jeweils ein Beispiel für Rituale weltlicher und geistlicher Devestitur im historischen Kontext des Investiturstreits (Königsabsetzungen und die Schandprozession zur Amtsenthebung Gregors VIII.) voran sowie - als Beispiel für eine Devestitur als Investitur - die Mönchsweihe, bei der das Ablegen der Kleidung die Weltabsage symbolisiere. In diesem Kontext arbeitet Kraß vier, auch für Devestiturszenen in den literarischen Umsetzungen signifikante Merkmale heraus. Demzufolge sind Devestiturrituale I. auf einen Bußakt zentriert, umfassen sie 2. den symbolischen Akt der Entkleidung, sind 3. Bußakt und Entkleidung an einen sozialen Statuswechsel geknüpft, und 4. lassen sich zwei Verlaufsperspektiven unterscheiden, „je nachdem ob die Devestitur den Gewinn oder Verlust einer Würde bewirkt" (S. 2 Io). Mit Blick auf die höfische Literatur differenziert Kraß zudem zwei Arten der Devestiturdarstellung: die Anlehnungen an die aus der Realgeschichte bekannten Rituale des Herrschaftsverzichts sowie die in den nachfolgenden Einzelanalysen ausschließlich behandelten Beispiele, in denen das lebensweltliche Ritual Modell stehe „für eine bestimmte Erzählfigur, die auf dem Individuationsprozeß des Protagonisten, in der Regel auf eine Identitätskrise bezogen ist" (S. 2 I0).

Wiederum präsentiert Kraß fünf Fallbeispiele, die, so will es der Verfasser zumindest einleitend, „in welchem Grad auch immer, auf den Gattungstyp der Legende bezogen sind“ (S. 30) und in denen es neben den Devestiturszenen vor allem auch um die Symbolik der eng daran gekoppelten Nacktheit geht: Im Orendel sei der graue Rock Zeichen der Erwähltheit des Protagonisten, dessen Königtum eine heiligmäßige Lebensform voraussetze. Die im Zustand prälapsaler Unschuld präsentierte und schamfreie Entkleidung/Nacktheit der weiblichen Protagonistin im Armen Heinrich markiere ein Opfer, ein Martyrium, „das [das Mädchen] zu einem Zeitpunkt, an dem es von Sünde noch nicht erfaßt wurde, ins Heil führen soll“ (S. 2 I 7 ). Im Iwein sei Nacktheit im Rahmen der Inszenierung einer Identitätskrise als Devestitur indes unmittelbar an den Verlust sozialer Identität geknüpft, während man bei der Nacktheit des Nackten Kaisers deutlich zwischen body natural and body political unterscheiden könne. ${ }^{2}$ Im Wolfdietrich schließlich stehe die Serie von fünf Devestiturszenen im Kontext von Begegnungen mit Gegenwelten. Die bei Kraß leider nur angesprochene, fünfte Devestitur Wolfdietrichs - das Ablegen der Rüstung zugunsten der Mönchskutte - ist meines Erachtens gerade im Hinblick auf deren Weiterführung im Epos klärungsbedürftig und hätte wohl mehr Beachtung verdient, denn die gleichsam doppelte, höfisch-ritterliche und monastische Identität in Gestalt der Rüstung über der Kutte des mönchgewordenen Helden (die so auch bei Ilsan im Rosengarten, bei Walther in Novalese im Chronicon Novalicense oder bei Ulrichs von Türheim Willehalm- und Rennewart-Figur vorkommt) scheint mir gerade aufgrund der daraus resultierenden diskursiven Spannungen ausgenommen interessant.

Nach Kraß ist an den behandelten Fallbeispielen deren Ambivalenz auffallend, darüber hinaus erscheinen Devestiturszenen einerseits als Folge schuldhaften Verhaltens, das den Verlust höfischen Ansehens mit sich bringe, andererseits können Entkleidungen auch den Aufstieg in eine höhere Stufe von Identität markieren, letztere würden „bereits das Signum von Heiligkeit" (S. 30) in sich tragen.

Implizierten die unter dem Begriff der „Spielregeln“ subsumierten vestimentären Codes der Einkleidung, Epiphanie und Auskleidung mit Blick auf

\footnotetext{
${ }^{2}$ Gerade der Vergleich von Armer Heinrich, Iwein und Nacktem Kaiser zeigt in diesem Zusammenhang überzeugend, daß Nacktheit nicht ausschließlich einen Verlust von Identität beinhaltet, wie es etwa noch Peter von Moos, „Das mittelalterliche Kleid als Identitätssymbol und Identifikationsmittel“. In: ders. (Hrsg.), Unverwechselbarkeit. Persönliche Identität und Identifikation in der vormodernen Gesellschaft. (Norm und Struktur 23) Köln u.a. 2004, S. I 23- I 46, veranschlagt, sondern durchaus auch einen Status des Übergangs markieren kann.
} 
die Konstitution höfischer Identität weitgehend eine affirmative Tendenz, so geben die „Spielfelder“ vestimentärer Codes, denen sich der dritte Hauptteil der Untersuchung zuwendet, deren transgressive Dimensionen zu erkennen. Unter Spielfeldern versteht der Verfasser „diejenigen Dispositive, die auf verschiedene Aspekte der Identität, nämlich des Standes (Maskerade), des Geschlechts (Travestie) und der Person (Kleidertausch) zielen und diese durch Grenzüberschreitungen in Szene setzen“ (S. 28 f.). Im parallelen Aufbau zu den vorangestellten Überlegungen zu den „Spielregeln“ bietet Kraß erneut jeweils einen Abriß der kulturgeschichtlichen Bedingungen der behandelten Einzelaspekte, dem eine chronologisch angeordnete Abfolge von literarischen Fallbeispielen nachsteht.

Als Spiel mit der sozialen Identität begreift Kraß dabei zunächst die Maskerade. Indem der Verkleidete eine Grenze überschreitet, erschließt er sich über seinen vertrauten Aktionsradius hinausgehende Spielräume und wird dadurch auch selbst zu einem anderen.

Obwohl Kleiderordnungen erst aus spätmittelalterlicher Zeit überliefert seien, haben - wie Kraß am Beispiel der Thematisierung von Standesgrenzen im Helmbrecht (Ritter/Bauer) und in Der gute Gerhard (Ritter/Kaufmann) anführt - „die normativen Kleidervorschriften Vorbilder in narrativen Texten, die das Verhältnis von Stand und Kleidung verhandeln“ (S. 3 I). Die literarischen Inszenierungen von Maskeraden sind meist in den Verkleidungsszenen des Brautwerbungsschemas zu finden, wodurch neben der Problematisierung von Standesidentität auch das Problem der Liebe zwischen Mann und Frau thematisiert wird. Dies führt Kraß zunächst am Beispiel der Tristan-Romane vor, wobei bei Eilhard von Oberg die Verkleidungsszenen in zunehmendem Maße verbunden seien „mit einer Reflexion auf das Problem von Wahrheit und Lüge, insbesondere auf die paradoxe Fähigkeit der Wahrheit, sich in der Maske der Lüge zur Geltung zu bringen“ (S. 252). Die poetologische Relevanz dieses Diskurses über Wahrheit und Lüge dränge sich dem Rezipienten geradezu auf. Bei Gottfried von Straßburg setzten die Maskeraden die Epiphanien des ersten Teils unter neuen Voraussetzungen fort, so würden Tristans Maskeraden als Pilger, Aussätziger und Büßer in unmittelbarem Wechselverhältnis mit Isoldes Rolle als Büßerin stehen, wodurch die Liebenden auch in ihrer Erniedrigung und in ihrem Leid vereint seien. Im Salman und Morolf komme es hingegen geradezu zu einem „Karussell von Maskeraden“ (S. 262), mit denen jedes Mal auch die Stände und somit auch die Identitäten gewechselt werden. „Als eine zynische Antwort auf die Tristanfigur“ (S. 265), die das Verhältnis von Identität und Maskerade invertiere, könne man Morolf betrachten, während sich die Maskerade bei Tristan als Zeichen seiner Identität offenbare, so erweise sich umgekehrt Morolfs Identität als Maskerade. Auch die Maskeraden in Strickers Der Pfaffe Amis, mit dem Kraß die Reihe seiner Fallbeispiele schließt, sind an das Handlungsmodell der List gebunden, wobei hier jedoch materielle Interessen im Vordergrund stehen würden. Als Fazit der behandelten Verkleidungsgeschichten kann Kraß überzeugend darlegen, wie verschiedene Aspekte - Maskerade und Identität, Schein und Sein - in einem dialektischen Verhältnis zueinander stehen. „Diese Einsicht verhandelt der Tristanroman am literarischen Phänomen der Fiktionalität, das Brautwerbungsepos Salman und Morolf am semiotischen Phänomen der Signifikation und der Schwankroman Der Pfaffe Amis am politischen Phänomen der Symbolik der Macht“ (S. 269).

Werden bei den Maskeraden Standesgrenzen überschritten, so kommt es im Rahmen eines Spiels mit der sexuellen Identität bei der Travestie zu einem Überschreiten von Geschlechtergrenzen innerhalb eines Standes, das die binäre Ordnung von Geschlechterdifferenz ebenso wie die von Heterosexualität ins Wanken geraten läßt. Der Auseinandersetzung einerseits mit dem Trojanerkrieg Konrads von Würzburg, in dem sich ein Ritter als Dame verkleidet, und andererseits dem Gürtel Dietrichs von Glesse, in dem sich umgekehrt eine Dame als Ritter ausgibt, stellt Kraß, ausgehend von den in diesem Kontext wohl obligaten Überlegungen insbesondere Judith Butlers und Thomas Laqueurs, einen Überblick über die physische und vestimentäre Ordnung beziehungsweise Differenzierung der Geschlechter in hochmittelal- 
terlicher Zeit voran. Der durchweg überzeugenden und detaillierten Auseinandersetzung insbesondere mit dem Text Konrads folgt der Befund, daß sowohl im Trojanerkrieg als auch im Gürtel „die Zuordnung von ,wahrem' und ,fiktivem' Geschlecht als Opposition von Körper und Kleidung in Szene gesetzt" (S. 298) werde, wobei sich die Richtung des Geschlechterwechsels als unerheblich erweise. Während Achill bei steter Präsenz der Geschlechterdifferenz in der Jocundille-Figur verdoppelt werde, konstituiere sich die Figur des Ritters Heinrich aus "der wechselseitigen Absorption einer männlichen und einer weiblichen Rolle“ (S. 300). An den Figuren des Heinrich und des Konrad werde die Abfolge derselben sozialen und erotischen Beziehungsformen durchgespielt wie im Trojanerkrieg: „Am Anfang steht die homosoziale Freundschaft, die im zweiten Schritt in die Nähe homosexueller Liebe rückt, um schließlich in die heterosexuelle Institution der Ehe überführt zu werden" (S. 30I). ${ }^{3}$

Als Spiel mit der personalen Identität zweier Freunde, die demselben Stand und demselben Geschlecht zugehören, begreift Kraß schließlich den dritten kategorialen Typ der Verkleidung, den Kleidertausch. Auf Grundlage einer Erörterung des Konzepts der geistlichen Freundschaft, „mit dem das Konzept der höfischen Freundschaft das zentrale Motiv des Herzenstauschs teilt" (S. 309), sowie einer Auseinandersetzung mit der geistlichen Ikonographie der Freundschaft richtet Kraß sein Augenmerk auf die vestimentären Zusammenhänge in literarischen Freundschaftsdarstellungen im Engelhard Konrads von Würzburg und im Großen Wolfdietrich. Gattungstypologisch gebe sich hier, so Kraß vorab, die Tendenz zu erkennen, daß der höfische Roman als Freundschafts- und Tauschsymbole eher Kleidung und Schmuck heranziehe, während die Heldenepik diesbezüglich Waffen und Rüstungen bevorzuge. Im Engelhard spiegele sich die seelische Einheit der Freunde in physischer Ähnlichkeit; die Spannung von Identität und Verschiedenheit, die die personale Union trage, werde in der Opposition von Körper und Kleidung umgesetzt: „Mit der Ähnlichkeit der Körper und der Differenz der Kleider sind die Voraussetzungen für jenes Betrugsmanöver geschaffen, mit dem Dietrich seinen Freund Engelhard aus der Todesgefahr befreit" (S. 327), denn durch den Kleiderwechsel werden vorübergehend auch die Identitäten gewechselt. „Dabei überträgt der Verfasser das Liebesmodell des Tristanromans auf die Union zwischen zwei Männern“ (S. 3I). Anders als in den Travestiegeschichten würden zudem die rivalisierenden Beziehungsformen im Engelhard auf eine Dreieckskonstellation projiziert, in der Ehe und Freundschaft konfrontiert werden. Ähnlich verhalte es sich auch im Wolfdietrich, freilich sei hier der durch Rüstungs- und Waffentausch sich vollziehende Identifikationsprozeß durch Angleichung erst am Ende des Heldenepos vollzogen: Wolfdietrich habe erst dann dauerhaft die soziale Rolle seines ehemaligen Rivalen und Freundes Ortnit angenommen, als er dessen Gebeine aus der Rüstung geschüttelt und sich diese angelegt, Ortnits Landesherrschaft angetreten und dessen Witwe geheiratet habe. Darüberhinaus müßten hier weitere Differenzen festgehalten werden, denn so läßt sich zwar mit Kraß ein vestimentär markierter Rollentausch festhalten (sofern man Rüstung und Waffen als Kleidung versteht), gleichwohl ist Freundschaft in der Heldenepik offensichtlich grundsätzlich anders in Szene gesetzt als in der höfischen Literatur. Es fehlt im Wolfdietrich die körperliche Ähnlichkeit der Freunde, auch spielt der Gedanke des „Einsseins im Herzen und der Seele“ keinerlei Rolle, vielmehr geht es um schlichtes und heldenepisch-trockenes Schwören von geselleschaft und Rache im Falle des Todes. Die Thematik der in beiden behandelten Texten auftretenden Dreiecksbeziehungen aufgreifend stellt $\mathrm{Kraß}$ in einem abschließenden Exkurs Überlegungen zur „triangulären Identifikation“ zur Diskussion. Hinterfragt wird dabei, inwieweit Girards Begriff des „triangulären Begehrens“ “

${ }^{3}$ Um wohl den Widerspruch von Tabuisierung und Legitimation der Thematisierung homoerotischen Begehrens in mittelalterlichen narrativen Texten aufzufangen, folgt der Analyse abweichend von der sonstigen Vorgehensweise der Untersuchung ein mit Blick auf die angesprochenen Texte gerichteter Exkurs zu den ,Schattengeschichten' in der Begrifflichkeit Stephen Greenblatts, die dieser am Beispiel der Shakespeare-Komödie Twelfth Night entwickelt; Stephen Greenblatt, „Dichtung und Reibung“. In: ders., Verhandlungen mit Shakespeare. Innenansichten der englischen Renaissance. Berlin I990, S. 66-9I und I62- I67.

${ }^{4}$ Nach René Girard, Figuren des Begehrens. Das Selbst und das Andere in der fiktionalen Realität. (Beiträge zur mimetischen Theorie 8) München I999, bes. S. I I-60. 
mit Blick auf die Figurenkonstellationen im Engelhard beziehungsweise Wolfdietrich durch den Begriff des „homosozialen Begehrens"s ersetzbar ist.

Das Schlußkapitel bündelt die Untersuchungsergebnisse in einem ersten Teilabschnitt zunächst mit der Frage nach charakteristischen „Spielplänen“, 6 nach denen sich ritterliche Identität auf Grundlage der sechs thematisierten vestimentären Kategorien (Investitur, Epiphanie, Devestitur, Maskerade, Travestie, Kleidertausch) ausbildet. Auf der Ebene der erzählten Geschichte (bistoire) sind dabei nach Kraß vier verschiedene Spielpläne unterscheidbar: Der Weg des Ritters zu sich selbst, die Bekehrung als der Weg des Ritters zum Göttlichen, die Liebeswerbung als Weg des Ritters zur Dame und schließlich die Freundschaftsprobe als Weg des Ritters zu einem anderen Ritter.

Weil sich im vestimentären Code die Ebenen des Erzählten und des Erzählens überkreuzen können, folgt in einem zweiten Schritt ein die Studie abschließender Ausblick auf das Motiv der Kleidung als poetologischer Metapher des Erzählens: „Texte scheinen als Textilien, insofern sie mit ihnen die Qualität eines Gewebes teilen; Textilien erscheinen als Text, insofern die beschriebenen Kleider aus den Worten des Beschriebenen bestehen“ (S. 339). Ausgehend von rezenten Thesen der Identitätsphilosophie (Paul Ricœur) und der Identitätspsychologie (Kenneth J. Gergen) zum Aspekt der „narrativen Identität“ macht Kraß mit Blick auf die Ebene des Erzählers (discours) darauf aufmerksam, daß „im Prozeß der narrativen Selbstidentifikation [...] eine rezeptionsästhetische und eine produktionsästhetische Dynamik“ zusammenspielen (S. 360). Als Beispiel für eine zeitgenössische pädagogische Reflexion über den moralischen Wert des höfischen Romans präsentiert Kraß erst die prominente Textpassage aus dem Welschen Gast Thomasins von Zerklaere (V. I I I - I I 26), in der mittels der Metapher der Einkleidung (der âventiure) auf den paradoxen Status höfischen Erzählens zwischen Wahrheit und Lüge verwiesen wird. Als Beispiele für entsprechende Verwendungen der Kleidermetaphorik in den höfischen Romanen selbst, in denen es um eine poetologische Selbstreflexion gehe, in der Dichtung als Kleid und der Dichter als Schneider metaphorisiert werden, führt Kraß sodann die Referenz des Chrétien de Troyes auf Macrobius im Kontext der Beschreibung von Erecs Krönungsmantel sowie den ebenfalls vieldiskutierten literaturkritischen Exkurs im Tristan Gottfrieds von Straßburg an. Schließlich greift Kraß den Titel seiner Studie im Hinblick auf die descriptio der schönen Kleider Flories im Wigalois auf, die Wirnt von Grafenberg betont als fiktiv, als „aus Worten gewirkt" (S. 374), darstellt; auf diese Weise sei die sichtbare Welt des Dichters und die imaginäre Welt der Dichtung (maere) unterscheidbar, sei Florie auch für den Rezipienten erkennbar letztlich „mit nichts anderem eingekleidet als mit den Worten des Dichters“ (ebd.).

'So unternimmt es Eve Kosofsky Sedgwick in ihrer Studie zu Dreiecksbeziehungen in englischen Romanen des I 8. und i 9. Jahrhunderts: Between Men. English Literature and Male Homosocial Desire. New York ${ }^{2}$ I992, auf die sich Kraß hier bezieht.

${ }^{6}$ Den Begriff des „Spielplans“ bestimmt Kraß aus drei Perspektiven: literaturtheoretisch in Anlehnung an Rainer Warnings Konzept der thematischen Rolle und Jurij Lotmans Konzept des literarischen Sujets sowie kulturtheoretisch mit dem Konzept des Übergangsritus nach Arnold van Gennep. 
Die Untersuchung besticht nicht allein durch ihre ausgenommen luzide Art der Darstellung, sondern auch durch ihre weitgehend überzeugenden, detaillierten und hochinteressanten Fallstudien. Hierin liegt paradoxerweise zugleich auch eine ihrer Schwächen, denn so beeindruckend die Studie in der Materialsammlung auch ist, die Arbeit an den Texten mutet doch zuweilen recht deskriptiv an, so daß der Leser am Ende manch eines Teilkapitels ob der Ergebnisse wenig überrascht, wenn nicht gar ratlos sein dürfte. Die kulturwissenschaftlich orientierte Anlage der Arbeit dürfte freilich in ihrem $\mathrm{Zu}$ sammenklang von moderner Theoriebildung und Auseinandersetzung mit der höfischen Literatur des Mittelalters für lange Zeit richtungsweisend bleiben.

Universität Zürich

Julia Zimmermann

Deutsches Seminar

Schönberggasse 9

$\mathrm{CH}-800$ I Zürich

j.zimmermann@ds.uzh.ch 